\title{
RELAÇÃO ENTRE MATURIDADE EM INOVAÇÃO SUSTENTÁVEL E O DESEMPENHO EMPRESARIAL
}

Data de submissão: 03/07/2018

Aceite: 25/09/2018

Edilson Bacinello ${ }^{1}$

Gérson Tontini

\section{RESUMO}

A Inovação Sustentável (IS) combina a criação de valor econômico, ecológico e social, tendo uma forte ligação com as dimensões do Triple Bottom Line (TBL) na perspectiva de longo prazo. Inovar, segundo essas dimensões, requer o desenvolvimento de novos instrumentos e modelos de gestão, tal como pode ser verificado nos modelos de Maturidade em Inovação Sustentável (MIS) para compreender a construção de determinada competência e evolução das empresas em determinada área. Nesse contexto, este estudo buscou verificar a relação existente entre a MIS e o Desempenho Financeiro e de Mercado (DFM) de empresas. A pesquisa, de cunho quantitativo, se pautou na Modelagem de Equações Estruturais (MEE) por meio do software Smart PLS. Os resultados apontam a existência de relação entre a MIS e o DFM, que pode ser explicada, em parte, pelas estratégias relacionadas à utilização de recursos e capacidades para geração de vantagem competitiva as empresas. Verificou-se, também, que a discussão teórica existente no tema relacionado à inovação com foco na sustentabilidade permanece inacabado, e que tanto os recursos como as capacidades organizacionais concorrem para poder explicar as relações existentes entre as ISs e o desempenho empresarial (DE).

Palavras-chave: Inovação Sustentável. Desempenho. Teoria Baseada em Recursos. Teoria das Capacidades Dinâmicas.

\footnotetext{
1 Possui graduação em Ciências Contábeis pela Universidade Federal de Rondônia, UNIR, mestrado em Administração pela UNIR e doutorado em Ciências Contábeis e Administração pela Universidade Regional de Blumenau, FURB. Porto Velho-RO. Brasil E-mail:edbaci@bol.com.br

2 Possui graduação em Engenharia Mecânica pela Universidade Federal de Santa Catarina, UFSC, mestradoem Engenharia Mecânica pela UFSC doutorado em Engenharia Mecânica pela Universidade Federal de Santa Catarina Sanduiche na Marquette University Us, UFSC/MARQUETTE. Blumenau -SC. Brasil. E-mail: tontini@furb.br
} 


\section{INTRODUÇÃO}

A inovação sustentável (IS) considera as perspectivas econômicas, sociais e ambientais das atividades organizacionais, proporcionando a geração de vantagem competitiva e melhoria do desempenho empresarial (DE). Os termos "IS" ou "Eco-inovação" têm sido amplamente definidos como o processo de desenvolvimento de novas ideias, comportamentos, produtos e processos que contribuam para a redução dos encargos ambientais ou ecológicos especificados nas metas de sustentabilidade empresarial (RENNINGS, 2000).

A Eco-inovação está fortemente atrelada às considerações ambientais (KEMP; PEARSON, 2008), enquanto a IS contempla uma maior amplitude, incluindo uma forte ligação com o Triple Bottom Line - TBL (HANSEN; GROBE-DUNKER; REICHWALD, 2009; BOCKEN et al., 2013; FROEHLICH, 2014). Este termo envolve o contexto das futuras gerações, ou seja, a perspectiva de longo prazo (HALL e VREDENBURG, 2003; CHARTER et al., 2008).

Os modelos de negócios sustentáveis que incorporam o TBL consideram uma ampla gama de interesses dos stakeholders, incluindo o meio ambiente e a sociedade (BOCKEN et al., 2013). Para os autores, esses negócios são importantes para conduzir e implementar a inovação corporativa para a sustentabilidade em processos comerciais, servindo como condutor principal da vantagem competitiva.

Inovar, segundo as dimensões do $T B L$, ainda não é a regra, até porque a inclusão das dimensões sociais e ambientais requer novos instrumentos e modelos de gestão empresarial que só recentemente começaram a ser desenvolvidos com mais intensidade (BARBIERI et al., 2010). Os autores relatam que o atendimento a essas dimensões torna o processo de inovação mais sofisticado e exigente, requerendo um maior esforço da organização para atender aos requisitos técnicos necessários à sua gestão.

Dentre os instrumentos de gestão empresarial, os modelos de Maturidade em Inovação Sustentável (MIS) vêm sendo utilizados como forma de compreender a construção de determinada competência nas organizações (DELAl, 2014). Os níveis de maturidade têm como objetivo compreender como ocorre a construção de determinada competência nas organizações, ou demonstrar como uma empresa está evoluída em determinada área (FOLLMANN, 2012).

Estudos relacionados ao desenvolvimento de MIS (HYNDS et al., 2014; GALVÃO, 2014) focaram, principalmente, nas questões ambientais/ecológicas da sustentabilidade. Por sua vez, Delai (2014) criou um modelo de estágios evolutivos em gestão da IS, demonstrando suas características e competências específicas, observando que inovar, segundo as dimensões da sustentabilidade, é imprescindível para avaliação da competitividade e do sucesso empresarial.

Embora alguns estudos tenham abordado perspectivas e conceitos específicos acerca das inovações relacionadas à sustentabilidade, existem poucas pesquisas com evidências empíricas sobre os conceitos referentes à IS. Alguns estudos verificaram as relações entre inovação e sustentabilidade (FOXON; PEARSON, 2008; BARBIERI et al., 2010; FROELICH, 2014; HYNDS et al., 2014), os estágios evolutivos da IS (DELAI, 2014) e a relação estratégica entre a inovação com enfoque sustentável e/ou IS e o DE (GUNDAY et al., 2011; GALVÃO, 2014; LOPEZ-VALEIRAS; GOMESCONDE; NARANJO-GIL, 2015; KNEIPP, 2016), chegando a resultados distintos. De acordo com Boons e Ludeke-Freund (2013), os resultados das pesquisas relacionadas a IS ainda não são conclusivos e tendem a negligenciar a necessidade das empresas em combinar uma proposta sobre a cadeia de valor na organização, considerando-a a montante e a jusante do modelo financeiro.

No mesmo sentido, identifica-se uma limitação nos modelos atuais de mensuração da MIS, dadas as diferentes perspectivas teóricas e empíricas utilizadas, que podem indicar uma ina- 
dequação dos resultados obtidos (DELAI, 2014). Segundo a autora, existe a necessidade de uma abordagem mais profunda que identifique os diferentes níveis em que essas mensurações ocorrem no contexto empresarial. Diante disso, o objetivo principal desse estudo é verificar a relação existente entre MIS e o desempenho financeiro e de mercado (DFM) das empresas.

A escolha da região amazônica para aplicação desse estudo justifica-se pela ausência de pesquisas que contemplem as iniciativas relacionadas à preservação e manutenção dos recursos e da biodiversidade, como também pelas complexas relações sociais que envolvem seu contexto histórico (PAS, 2008). Essa perspectiva considera a exploração de recursos e capacidades regionais, sua valorização a partir da agregação de valor e criação de oportunidades objetivas para geração de emprego e renda.

A investigação proposta pretende contribuir para uma maior compreensão das estratégias que envolvem as ISs, a partir da utilização de um modelo de MIS para verificar sua relação com o DFM das empresas. Busca também discutir acerca das possíveis abordagens teóricas que envolvem o tema.

O estudo está estruturado por esta introdução e mais quatro seções. Na seção 2, é feita uma revisão da literatura sobre os tópicos referentes a IS, seu contexto estratégico, a utilização dos recursos e capacidades como fonte de vantagem competitiva e estudos similares. A seção 3 compreende a apresentação dos procedimentos metodológicos utilizados. Na seção 4 são apresentados e discutidos os resultados. Por fim, na seção 5 , são feitas as considerações finais.

\section{FUNDAMENTAÇÃO TEÓRICA}

Nesse capítulo será feita uma revisão teórica sobre IS, seu contexto estratégico, a utilização dos recursos e capacidades relacionados a obtenção de vantagem competitiva e estudos similares.

\subsection{Inovação Sustentável}

A complexidade dos fatores que influenciam as decisões relacionadas a inovação devem ser atreladas a um arcabouço teórico que integre as abordagens neoclássicas e evolutivas, e que verifique o papel específico dos instrumentos regulatórios relacionados ao tema (RENNINGS, 2000). Na visão do autor, a integração dos aspectos ecológicos, sociais e econômicos do desenvolvimento sustentável é altamente útil para a abertura de pesquisas sobre inovação, mudanças sociais e institucionais.

Eco-eficiência ou eco-inovação implica a redução dos impactos ambientais (KEMP; PEARSON, 2008), tentando alinhar o uso de recursos ao longo de todo o ciclo de vida do produto para corresponder à capacidade de suporte da Terra, e ao mesmo tempo busca oferecer produtos a preços competitivos, satisfazer as necessidades humanas e trazer qualidade de vida (HELLSTROM, 2007). Para os autores, esse termo diz respeito a melhorias incrementais nos processos, tais como a substituição de componentes ou incorporação de fatores a produtos já existentes, utilizando alternativas mais amigáveis ambientalmente.

A IS vai além de eco-inovação, porque inclui objetivos sociais ligados de forma mais clara ao processo holístico de longo prazo do desenvolvimento sustentável, considerando os objetivos de curto e longo prazo (CHARTER et al., 2008). A IS se refere a um processo que considera a sustentabilidade no âmbito financeiro, social e ambiental (HANSEN; GROBE-DUNKER; REICHWALD, 2009; BOONS et al., 2013). Integra sistemas para geração de ideias por meio de pesquisa e desenvolvimento (P\&D) e comercialização de produtos, serviços e tecnologias, bem como novos modelos de organização empresarial (CHARTER; CLARK, 2007; CHARTER et al., 2008). 
Charter e Clark (2007) definem a inovação orientada à sustentabilidade como a criação de novos espaços no mercado, produtos, serviços ou processos orientados por questões sociais, ambientais ou de sustentabilidade. Segundo os autores, tal como acontece com a inovação geral, existe um reconhecimento emergente de que a IS não diz respeito apenas a novos conceitos, mas sim à comercialização de tecnologias, produtos, serviços e empreendedorismo, assim como sobre a adoção de novos processos e sistemas a nível social.

De acordo com Boons et al. (2013), a IS tem diferentes significados em diferentes contextos, tais como economias de consumo, economias emergentes e economias de base da pirâmide. De acordo com os autores, qualquer alteração significativa da lógica econômica dominante envolve, necessariamente, a aplicação de novos negócios nos modelos sociais por parte de atores que procuram promover ideias. Relatam ainda que isso pode levar a diferentes tipos de inovações e práticas diferentes sobre as proposições de valor e reflexão sobre as verdadeiras necessidades dos cidadãos, definidas em categorias que não sejam puramente econômicas.

A IS está diretamente relacionada à introdução (produção, assimilação ou exploração) de produtos, processos produtivos, métodos de gestão ou negócios, novos ou significativamente melhorados para a organização e que traz benefícios econômicos, sociais e ambientais, comparados com alternativas pertinentes, nos quais são esperados benefícios significativos ou não negligenciáveis no TBL (BARBIERI et al., 2010). Para os autores, as ISs consideram uma lista ampla de partes interessadas secundárias, como a comunidade local e grupos ativistas de várias causas, que levam ao crescimento do movimento pela responsabilidade social da empresa.

Embora o termo IS tenha sido amplamente utilizado, existe certa limitação em sua definição (BOONS et al., 2013). De acordo com os autores, o conceito oferece uma ferramenta analítica que permite avaliar a interação entre os diferentes aspectos das empresas, combinando a criação de valor econômico, ecológico e social. Dessa forma, a criação de valor depende da habilidade das empresas em construir uma reputação positiva ao longo do tempo por meio da implementação de inovações (HART; MILSTEIN, 2004).

Nesse contexto, formula-se a primeira hipótese (H1): As ISs conduzem a diferentes tipos de inovações que possibilitam a criação valor para as empresas.

\subsection{Contexto Estratégico da Inovação Sustentável}

Hall e Vredenburg (2003) defendem a necessidade de uma estratégia que integre os objetivos de inovação e do desenvolvimento sustentável. Os autores relatam que a IS deve orientarse para o mercado a partir da inclusão dos princípios do desenvolvimento sustentável, buscando tanto a incorporação das restrições oriundas das pressões sociais e ambientais como uma visão que considere as futuras gerações em uma perspectiva de longo prazo.

A IS pode estar relacionada a uma postura estratégica e sistemática da empresa no que se refere aos aspectos econômicos, sociais e ambientais, e não apenas a ações isoladas, como o desenvolvimento de novos processos e produtos ambientalmente corretos (SCHALTEGGER; LÜDEKE-FREUND; HANSEN, 2011; HANSEN; GROBE-DUNKER; REICHWALD, 2009; HYNDS et al. 2014; KNEIPP, 2016). Para os autores, uma orientação contínua para a inovação com foco na sustentabilidade requer modificações no modelo de negócios das empresas a fim de permitir o gerenciamento de atividades sociais e ambientais de forma sistemática.

O valor sustentável deve ter o objetivo estratégico de evitar que as empresas diminuam seus lucros e o valor gerado aos acionistas, uma vez que as ações relacionadas à sustentabilidade e à criação de valor devem estar diretamente relacionadas (HART; MILSTEIN, 2004; BOONS; LU- 
DEKE-FREUND, 2013). Segundo os autores, as empresas devem criar produtos e serviços futuros por meio do desenvolvimento de capacidades e tecnologias que proporcionem o crescimento da organização, mantendo o desempenho dos negócios.

De acordo com Oksanen e Hautamaki (2015), as questões referentes à IS são complexas e, para que ela possa ocorrer de fato, requer uma ampla cooperação que envolve muitos atores. Para os autores, essas inovações são pautadas em três definições características estratégicas:

1) contribuir para o bem-estar sustentável: criar soluções em que todos sejam capazes de encontrar seu papel dentro da rede de resoluções de problemas e, assim, criar a vantagem competitiva;

2) ser sistêmica: estar relacionada à mudança tecnológica e cultural, construindo o núcleo das estratégias nacionais de inovação para serem implementadas no contexto da organização, e

3) ser inclusiva: apoiar a sabedoria coletiva e a colaboração em massa, em que os cidadãos tenham o direito de ser criativos e possam contribuir para melhorias nos serviços e produtos.

As práticas de gestão da IS contribuem para a implementação das estratégias que meIhorem a posição da empresa no futuro, pressupondo a mobilização de recursos, o desenvolvimento das habilidades dos funcionários, comunicação flexível, informações compartilhadas, investimento em P\&D e a busca de fontes de informações externas que possam favorecer o aumento do conhecimento (GALVÃO, 2014). O autor infere que o maior grau da mobilização de recursos e capacitações propicia a aprendizagem organizacional, necessária para promover mudanças e inovações em processos e produtos sustentáveis.

Diante disso, elabora-se a segunda hipótese (H2): As ISs promovem um melhor posicionamento estratégico nas práticas sustentáveis implementadas pelas empresas.

\subsection{Recursos e Capacidades Relacionados a Vantagem Competitiva}

Do ponto de vista empresarial, existe um amplo consenso de que os desafios da sustentabilidade oferecem um potencial significativo para inovações e oportunidades para geração de vantagem competitiva (HANSEN; GROBE-DUNKER; REICHWALD, 2009). Segundo os autores, dois argumentos apoiam esta visão:

1) novos regulamentos sociais e ambientais aumentam a pressão pela capacidade de inovação;

2) novas oportunidades de negócios, oriundas, principalmente, da redução de custos por meio do aumento da eficiência, da redução dos riscos, da fiabilidade do planejamento, legitimidade, atração de novos segmentos de clientes e desenvolvimento de novos produtos e segmentos de negócios.

As empresas buscam reter recursos como forma de gerar valor próprio aos negócios por meio da associação e combinação de recursos valiosos que dinamizam novas competências e capacidades de inovação distinta de alto valor agregado que podem gerar vantagem competitiva (JANG, 2013). De acordo com o autor, a capacidade de criação/inovação é muito importante para reforçar o núcleo de recursos empresariais cuja dinâmica resulte em novas combinações e novos recursos para assegurar uma vantagem duradoura sobre a concorrência.

De acordo com a Teoria Baseada em Recursos (TBR) das empresas (GRANT, 1991), as empresas utilizam os recursos disponíveis para obterem vantagens na implementação de estratégias no mercado (WERNERFELT, 1984; BARNEY; ARIKAN, 2001). Assim, as vantagens relativas de uma empresa, ou dotação de recursos, dependem dos fatores de mercado que sustentam diferenças em termos de eficiência nos resultados organizacionais (LOCKHET, 2005).

No âmbito estratégico, as relações existentes entre recursos, concorrência e rentabilidade (BARNEY, 1986) incluem a análise da imitação competitiva e a adequação dos retornos às inovações pelas quais o processo de acumulação de recursos pode sustentar a vantagem compe- 
titiva (GRANT, 1991). Essa vantagem pode ocorrer quando se implementa um valor que seja raro, não simultaneamente utilizada pelos concorrentes e quando outras empresas sejam incapazes de duplicar ou substituir (BARNEY, 1991).

Para as empresas adquirirem um certo recurso, elas dependem de já terem desenvolvido outros recursos anteriores ou possuírem uma determinada capacidade (HART, 1995). Segundo o autor, essa interconectividade consiste na dependência de caminho (sequência específica de acumulação) e enraizamento (tornar mais difícil o desenvolvimento de um novo recurso), como forma de compartilhar o desenvolvimento sustentável acelerando o desenvolvimento de recursos e capacidades empresariais.

Em analogia com a TBR, Teece e Pisano (1994) indicam a necessidade de um paradigma expandido para explicar com a fonte de vantagem competitiva é obtida e mantida. Afirmam que as "Capacidades Dinâmicas" (CD) enfatizam o caráter mutável do meio ambiente e o papel fundamental do gerenciamento estratégico na adaptação, integração e reconfiguração das habilidades internas e externas relativas aos recursos e competências funcionais do ambiente organizacional.

O termo "dinâmico" refere-se ao caráter mutante do meio ambiente em que certas respostas estratégicas são necessárias para determinar o ritmo da inovação acelerada e a natureza da concorrência futura dos mercados (TEECE; PISANO, 1994). Ainda segundo os autores, as "capacidades" enfatizam o papel fundamental da gestão estratégica em adaptar, integrar e reconfigurar as habilidade organizacionais (internas e externas), os recursos e competências funcionais.

Teece, Pisano e Shuen (1997) inferem que as CD indicam as fontes e os métodos de criação de riqueza para as empresas que operam em ambientes de rápida mudança tecnológica, em que a vantagem competitiva é moldada pelas posições de ativos (específicos), ou seja, recursos. A adaptação do conjunto destes recursos indicam o caminho evolutivo (experiência organizacional) que a empresa herdou. Para os autores, as CD se referem à capacidade de integrar, construir e reconfigurar competências internas e externas em ambientes de rápida mudança, refletindo a capacidade de uma organização alcançar novas e inovadoras formas de vantagem competitiva, dadas as dependências do caminho e as posições do mercado.

McKelvie e Davidson (2009) argumentam que as CD podem gerar ideias, provocar ruptura no mercado, desenvolver produtos, serviços e processos inovadores. Afirmam que, embora diversas observações tenham afirmado que as $C D$ sejam uma fonte de vantagem competitiva, existe pouco conhecimento sobre como o acesso aos recursos as afetam. Nesse sentido, Eisenhardt e Martin (2002) indicam que as CD se referem às "melhores práticas" nas empresas e seu valor para a vantagem competitiva reside nas configurações de recursos e não nas próprias capacidades.

Surge, assim, a terceira hipótese (H3): As bases teóricas da TBR e das CD podem auxiliar na explicação da vantagem competitiva oportunizada pelas IS.

\subsection{Estudos Anteriores sobre Inovação Sustentável}

Froehlich (2014) analisou o desenvolvimento da capacidade de inovação como meio para alavancar a sustentabilidade empresarial, utilizando a IS para ampliação da capacidade de inovação nos pilares econômico, ambiental e social da sustentabilidade empresarial. Propõe como principal resultado da pesquisa, a necessidade de desenvolvimento efetivo da capacidade de IS para alavancar o processo de sustentabilidade empresarial.

Galvão (2014) buscou entender a influência das práticas de gestão socioambiental para o desempenho da eco-inovação empresarial, identificando uma influência positiva das práticas voltadas para recursos e capacidades de inovação ambiental em processos de desempenho eco -inovador das empresas. 
Delai (2014) propôs um modelo de MIS para análise do padrão evolutivo na gestão da IS e gestão da sustentabilidade. A autora definiu quatro estágios da inovação (operacional, estratégico hard, estratégico soft e melhoria estratégica), concluindo que a evolução da gestão da inovação e da sustentabilidade mostrou-se linear (estágios de forma sequencial), enquanto no caso da gestão da IS os resultados demonstraram ser não-lineares.

Hynds et al. (2014) desenvolveram um modelo de MIS para avaliar e orientar organizações de P\&D na criação de produtos e serviços que impulsionem o crescimento das ISs, principalmente relacionados aos aspectos ambientais da sustentabilidade. Verificaram a existência de correlação entre as práticas sustentáveis e a margem operacional, indicando que as empresas devem estabelecer métricas claras e relevantes para rastrear seu progresso com base em necessidades estratégicas específicas relacionadas a sustentabilidade.

Kneipp (2016) analisou a relação da gestão estratégica da IS com o modelo de negócios e o desempenho de empresas industriais brasileiras e espanholas. Com base na análise de duas dimensões (postura estratégica e práticas de IS), a autora demonstrou que a gestão estratégica da IS está relacionada com o modelo de negócios e o DE.

Nese contexto, elabora-se a quarta hipótese (H4): A MIS auxilia no processo de gestão da sustentabilidade, exercendo influência no DE.

\section{METODOLOGIA}

A pesquisa, de cunho quantitativo, se pautou no envio/aplicação de um questionário fechado contendo cinco questões referentes ao nível de MIS nas empresas ((BARBIERI et al., 2010; KNEIPP, 2016), dispostas em uma escala de cinco pontos, que vai de 1 (inexistência de práticas e padrões); 2 (informal ou em fase de implantação); 3 (formalmente estabelecido); 4 (estabelecido e sistêmico) e 5 (estabelecido, sistêmico e otimizado). Verificou-se, também, outras cinco questões referentes ao DFM (PELHAM; WILSON, 1996; GUNDAY et al., 2011; CHENG; YANG; SHEU, 2014), dispostas em uma escala likert de 5 pontos, que variou entre 1 (muito baixo), 2 (inferior), 3 (na média), 4 (elevado) e 5 (muito alto) em relação aos concorrentes.

Os dados foram analisados por meio da Modelagem de Equações Estruturais (MEE) pelo método Partinal Least Squares (PLS), ou Mínimos Quadrado Parciais. Esse método permite verificar, dentre outros fatores, a Variância Média Extraída (AVE), o Alpha de Cronbach (AC), a Confiabilidade Composta (CC), a Validade Discriminante (VD) e o Tese T de Student. Hair et al. (2014) tratam que a MEE é uma classe de técnicas multivariadas que combinam aspectos de análise fatorial e regressão, permitindo examinar simultaneamente os relacionamentos entre Variáveis Observadas e Variáveis Latentes (VL), bem como entre VL.

A Modelagem PLS utiliza dados disponíveis para estimar as relações do caminho no modelo com o objetivo de minimizar os termos de erro (isto é, a variância residual) das construções endógenas, ou seja, as estimativas dos coeficientes que maximizam os valores $\left(R^{2}\right)$ das construções endógenas (HAIR et al., 2014). Segundo os autores, o PLS tem como objetivo desenvolver uma teoria e explicar sua variância (previsão das construções).

O PLS estima uma série de regressões de mínimos quadrados que decorre da estimação iterativa dos parâmetros (LEE et al., 2011). Esse método funciona de forma eficiente em pequenas amostras de modelos complexos e pode ser aplicado em uma grande variedade de situações de pesquisa (HAIR et al., 2014). 


\subsection{Amostra de Pesquisa}

A pesquisa foi realizada entre os meses de fevereiro a junho de 2016, por meio de um banco de dados que continha 975 entidades relacionadas a indústria, obtido junto à Federação das Indústrias do Estado de Rondônia (FIERO, 2016).

Sequencialmente foram coletados os endereços eletrônico e/ou pessoal via linkedln das para envio de 532 questionários aos gerentes/responsáveis das empresas. Ao final da coleta haviam sido obtidos 63 questionários, que, após exclusão de 5 respostas (preenchidas erroneamente), resultou em uma amostra final de 58 respondentes, número considerado adequado para a MEE - PLS (HAIR et al., 2014).

\subsection{Variáveis de Pesquisa}

As variáveis de IS (BARBIERI et al., 2010; KNEIPP, 2016) se pautaram nas Ações voltadas a novos processos (ISNPR), Ações voltadas a novos produtos e/ou serviços (ISNPS), Ações relacionadas a novos métodos de gestão ou negócios (ISNMGE), Gestão de processos tecnológicos ou pesquisas organizacionais (ISPTPO) e Processo para criação de valor com ISs (ISCVI).

As variáveis de DFM empresarial (PELHAM; WILSON, 1996; GUNDAY et al., 2011; CHENG; YANG; SHEU, 2014) se embasaram nos indicadores Ativo Total (DESAT), Lucro (DESL), Satisfação dos Clientes (DESCS), Participação no Mercado (DESPM) e Valor Agregado do Produto/Serviço (DESVAPS).

\subsection{Análise dos dados}

De acordo com a MME - PLS, devem ser seguidos os seguintes passos/procedimentos para a análise do construto proposto na pesquisa: verificação do AC, CC, AVE, VD, o Teste "T" de Student e, por fim, a avaliação do modelo estrutural (RINGLE; SILVA; BIDO, 2014; HAIR et al., 2014).

O AC é um estimador imparcial da correlação entre as respostas de um questionário, calculado a partir da variância dos itens avaliados (CRONBACH, 1951). O valor mínimo aceitável é 0,70 , pois, abaixo desse valor, a consistência interna da escala utilizada é considerada baixa (STREINER, 2003).

A CC varia entre 0 e 1 e é geralmente interpretada da mesma maneira que o AC. Especificamente, valores entre 0,60 a 0,70 são aceitáveis em pesquisas exploratórias, e em estágios avançados de pesquisa pode ultrapassar 0,90 (NUNNALLY; BERSTEIN, 1994). Segundo os autores, a CC abaixo de 0,60 indica falta de confiabilidade de consistência interna do construto.

De acordo com Hair et al. (2014, p. 103), "a AVE é o valor médio das cargas quadradas dos indicadores associados à construção, ou seja, a soma do quadrado dos carregamentos divididos pelo número de indicadores". Para os autores, a AVE é equivalente à comunidade de um construto, em que um valor de 0,50 ou maior indica que, em média, o modelo explica mais do que metade da variância de seus indicadores.

A VD é a medida em que uma construção é verdadeiramente distinta de outras construções por padrões empíricos, implicando ser a única que captura fenômenos não representados por outras construções no modelo (HAIR et al., 2014).

O Teste " $\mathrm{T}$ " busca testar a hipótese de diferença entre duas médias sob a hipótese nula de que elas são iguais, tendo a capacidade de detectar diferenças significativas entre conjuntos de dados com médias similares (HAIR et al., 2014). Segundo os autores, os valores do teste " $T$ " iguais ou acima de 1,96, com significância de 0,05, são aceitáveis. 
$\mathrm{Na}$ avaliação do modelo estrutural, o coeficiente de Pearson $\left(\mathrm{R}^{2}\right)$ avalia a porção da variância das variáveis endógenas (RINGLE; SILVA; BIDO, 2014), sendo classificado como de efeito pequeno $(0,02)$, com efeito médio $(0,13)$ e com grande efeito $(0,26)$ nos resultados (COHEN, 1998). Verifica-se também o $R^{2}$, a Relevância Preditiva $\left(Q^{2}\right)$ que indica o quanto o modelo se aproxima do que se esperava dele e o Tamanho de Efeito $\left(\mathrm{f}^{2}\right)$ que permite avaliar a contribuição de uma construção (HAIR et al., 2014).

\section{RESULTADOS E DISCUSSÃO}

A verificação da consistência dos componentes dos questionários por meio do cálculo do AC, CC e AVE indicou os seguintes resultados:

Tabela 1: Estatísticas de confiabilidade e Variância Média

\begin{tabular}{c|c|c|c|c|c}
\hline Dimensões & N & Itens & $\begin{array}{c}\text { Alfa de } \\
\text { Cronbach } \\
\text { (AC) }\end{array}$ & $\begin{array}{c}\text { Confiabilidade } \\
\text { Composta (CC) }\end{array}$ & $\begin{array}{c}\text { Variância Média } \\
\text { Extraída (AVE) }\end{array}$ \\
\hline $\begin{array}{c}\text { Maturidade em Inovação Sustentável } \\
\text { (MIS) }\end{array}$ & 58 & 5 &, 934 &, 950 &, 792 \\
\hline $\begin{array}{c}\text { Desempenho Financeiro e de Mercado } \\
\text { (DFM) }\end{array}$ & 58 & 5 &, 815 &, 871 &, 575 \\
\hline
\end{tabular}

Fonte: Dados de Pesquisa - software Smart PLS

A Tabela 1 apresenta o número total de entrevistas ( $N=58)$, o número de itens de cada VL $(5)$, os resultados ao AC referente a VL em MIS $(0,934)$ e o DFM $(0,815)$, superiores a 0,7 (STREINER, 2003), indicando alta correlação entre as variáveis (CRONBACH, 1951), a CC (MIS: ,950 e DFM: , 872) considerados aceitáveis e em estágio avançado de pesquisa (NUNNALLY; BERSTEIN, 1994) e AVE (MIS: , 792 e DFM: , 575) assinalando que, em média, o modelo explica mais do que metade da variância de seus indicadores (HAIR et al., 2014).

A VD (método das cargas cruzadas) demonstrou que a construção de cada VL é verdadeiramente distinta da outra (HAIR et al., 2014), implicando as cargas fatorias se situarem no âmbito de suas respectivas dimensões, conforme Tabela 2 .

Tabela 2: Validade Discriminante

\begin{tabular}{c|c|c|c|c|c}
\hline Variáveis & DFM & MIS & Variáveis & DFM & MIS \\
\hline DESAT & $\mathbf{0 . 8 4 6}$ & 0.569 & ISCVI & 0.556 & $\mathbf{0 . 9 2 0}$ \\
\hline DESLC & $\mathbf{0 . 7 2 5}$ & 0.411 & ISNMGE & 0.526 & $\mathbf{0 . 9 3 7}$ \\
\hline DESPM & $\mathbf{0 . 7 8 0}$ & 0.465 & ISNPR & 0.491 & $\mathbf{0 . 8 7 3}$ \\
\hline DESSC & $\mathbf{0 . 6 8 0}$ & 0.340 & ISNPS & 0.592 & $\mathbf{0 . 9 1 0}$ \\
\hline DESVAPS & $\mathbf{0 . 7 4 8}$ & 0.419 & ISPTPO & 0.457 & $\mathbf{0 . 8 0 4}$ \\
\hline
\end{tabular}

Fonte: Dados de Pesquisa - software Smart PLS

O Teste "T" de Student, obtido pelo módulo bootstrapping (Smart PLS), indicou na MIS e no DFM valores bem superiores a 1,96 para o nível de significância de 0,05 , demonstrando, assim, a diferença entre duas médias sob a hipótese nula (HAIR et al., 2014), conforme demonstrado na tabela 3. 
Tabela 3: Teste "T" e P-value

\begin{tabular}{c|c|c|c|c|c}
\hline Variáveis & "T" & P-value & Variáveis & "T" & P-value \\
\hline DESAT & 23.567 & 0.000 & ISCVI & 50.723 & 0.000 \\
\hline DESLC & 10.059 & 0.000 & ISNMGE & 77.857 & 0.000 \\
\hline DESPM & 14.711 & 0.000 & ISNPR & 27.877 & 0.000 \\
\hline DESSC & 6.863 & 0.005 & ISNPS & 43.400 & 0.000 \\
\hline DESVAPS & 12.753 & 0.000 & ISPTPO & 12.795 & 0.000 \\
\hline
\end{tabular}

Fonte: Dados de Pesquisa - software Smart PLS

Os Valores de $Q^{2}$ (avaliação para a relevância preditiva) se mostraram positivos e superiores a zero $(0,171)$, indicando que o modelo se aproxima do que se esperava dele. $O f^{2}$ não foi calculado pelo fato de não terem sido incluídos e/ou excluídos construtos no modelo (HAIR et al., 2014).

No modelo completo do PLS é possível verificar o coeficiente de caminho Beta $(\beta)$ da relação existente entre a MIS e o DFM $(0,593)$. No caso específico desse estudo, o $R^{2}$ foi de 0,351 , ou seja, a MIS explica em torno de $35,1 \%$ do DFM, assinalando um grande efeito nos construtos (COHEN, 1998). Dessa forma, o modelo demonstra a capacidade de interação interativa entre os parâmetros (LEE et. al., 2011), conforme Figura 1.

Figura 1: Modelo Estrutural Completo

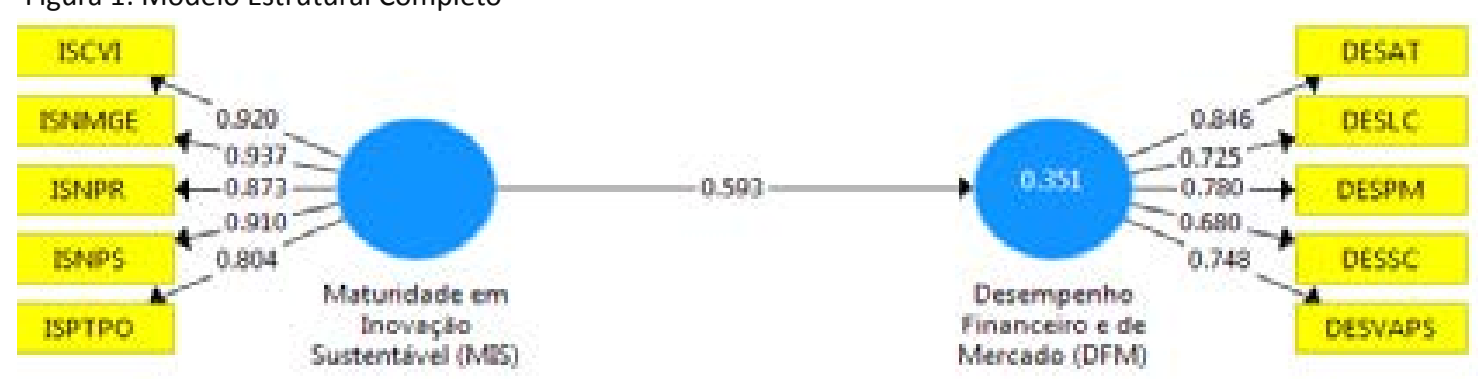

Fonte: Dados de Pesquisa - software Smart PLS

Os resultados obtidos no PLS-MEE corroboram, de forma geral, com os níveis médios de MIS encontrados na pesquisa, sendo que, da amostra total, quatorze empresas que apresentaram níveis mais elevados de maturidade (4 e 5) também indicaram os melhores resultados no DFM. Por sua vez, as oito empresas que assinalaram os menores níveis de MIS (1 e 2) também tiveram um menor DFM. Verificou-se ainda que das dezenoves empresas que apresentaram o nível médio de MIS (3), sete tiveram um nível médio de DFM, enquanto que as demais (doze) se situaram em uma escala superior de DFM. As demais empresas (dezessete) alternaram entre os melhores, médios e menores resultados de MIS e DFM.

Os resultados permitem indicar que os negócios sustentáveis que incorporam o TBL (HANSEN; GROSSE-DUNKER; REICHWALD, 2009; BOCKEN et al., 2013; FROEHLICH, 2014) relacionados aos instrumentos de gestão (BARBIERI et al., 2010) e ao desempenho financeiro empresarial (GUNDAY et al., 2011; LOPEZ-VALEIRAS; GOMES-CONDE; NARANJO-GIL, 2015) ainda não são conclusivos (BOONS; LUDEKE-FREUND, 2013), tanto pela limitada base teórica sobre IS (FOXON; PEARSON, 2008; BARBIERI et al., 2010) como pelos poucos estudos empíricos sobre o tema (GALVÃO, 2014; DELAI, 2014; HYNDS et al., 2014; KNEIPP, 2016).

Com base nesse estudo, o desenvolvimento dos modelos de MIS no contexto estratégico das organizações (HYNDS et al., 2014; GALVÃO, 2014; DELAI, 2014; KNEIPP, 2016) pode auxiliar, dentre outros fatores, em melhorias incrementais nos processos (HELLSTROM, 2007; CHARTER et al., 2008), criação de novos espaços no mercado (CHARTER; CLARK, 2007), produtos e serviços 
(RENNINGS, 2000; BOONS et al., 2013; BARBIERI et al., 2010), integrados em sistemas para geração de ideias, pesquisas em P\&D e tecnologias (CHARTER; CLARK, 2007; CHARTER et al., 2008) que combinem a criação de valor econômico, ecológico e social. Assim, aceita-se H1.

As empresas devem incorporar as restrições oriundas das pressões sociais e ambientais (KEMP; PEARSON, 2008) e o desenvolvimento de novos processos e produtos ambientalmente corretos (SCHALTEGGER; LÜDEKE-FREUND; HANSEN, 2011; HANSEN; GROBE-DUNKER; REICHWALD, 2009; KNEIPP, 2016) como forma de evitar uma redução nos lucros e no valor criado aos acionistas, mantendo o desempenho dos negócios e um melhor posicionamento estratégico (VANCONCELOS, 2001; HART; MILSTEIN, 2004; BOONS; LUDEKE-FREUND, 2013) na perspectiva de longo prazo (HALL; VREDENBURG, 2003). Dessa forma, a estratégia competitiva relacionada à criação de produtos ou processos (OKSANEN; HAUTAMAKI, 2015) pode mobilizar recursos, habilitar funcionários e permitir que as empresas invistam em informações de maior conhecimento (GALVÃO, 2014). Nesse sentido, aceita-se H2.

A potencial geração de vantagem competitiva oriunda das ISs oportuniza novos regulamentos sociais e ambientais e novas oportunidades de negócios (HANSEN; GROBE-DUNKER; REICHWALD, 2009), envolvendo uma ampla cooperação de vários atores (OKSANEN; HAUTAMAKI, 2015). Esses negócios reforçam o núcleo de recursos cuja dinâmica resulte em novas combinações e novos recursos (JANG, 2013) que assegurem uma vantagem duradoura para as empresas (BARNEY, 1991; HART; MILSTEIN, 2004).

Nesse contexto, a TBR, quando da implementação de estratégias no mercado (WERNERFELT, 1984; BARNEY; ARIKAN, 2001) e de vantagens relativas em termos de eficiência (LOCKHETT, 2005), expressa as relações existentes entre recursos, concorrência e rentabilidade (BARNEY, 1991) que podem sustentar a vantagem competitiva (GRANT, 1991). Tal vantagem pode ocorrer quando se implementa valor, raridade, dificuldade de imitação e não utilização simultânea dos concorrentes (BARNEY, 1991) e pode envolver questões relacionadas à sustentabilidade (HART, 1995; SHRIVASTAVA; HART, 1995; RUSSO; FOUTS, 1997; SHARMA; VREDENBURG, 1998).

Complementarmente, verifica-se a necessidade da expansão teórica para explicar a obtenção e manutenção de vantagem competitiva, indicando as fontes e os métodos para a criação de riqueza por meio tanto da posição dos ativos ou recursos (TEECE; PISANO, 1994; TEECE; PISANO; SHUEN, 1997) como também da geração de ideias, desenvolvimento de produtos, serviços e processos inovadores (MCKELVIE; DAVIDSON, 2009). A forma pela qual o acesso aos recursos afetam as empresas faz com que a vantagem competiva resida nas configurações dos recursos (EISENHARDT; MARTIN, 2002), embora as capacidades das empresas também possuam o potencial de geração dessa vantagem (TEECE; PISANO; SHUEN, 1997). Assim, aceita-se H3.

A capacidade das empresas se envolverem no processo de sustentabilidade em seus modelos de negócios (FROEHLICH, 2014; HYNDS et al., 2014) faz com que a gestão estratégica da MIS (GALVÃO, 2014; DELAI, 2014) apresente melhores resultados de desempenho financeiro e de mercado para as empresas que se posicionam nos melhores níveis de MIS e de forma contrária para as que se situem nos menores níveis (KNEIPP, 2016). Dessa forma, aceita-se H4.

Com base nessas afirmações, verifica-se que, por meio da gestão proveniente dos modelos de MIS, é possível verificar de que forma são desenvolvidas e gerenciadas as ISs, proporcionando uma fonte de vantagem competitiva e desempenho superior às empresas. Assim, a complementariedade na utilização dos recursos e capacidades empresariais oportunizam a criação de valor aos negócios sustentáveis. 


\section{CONSIDERAÇÕES FINAIS}

O objetivo desse estudo consistiu em verificar a relação existente entre MIS e DFM das empresas. Os resultados indicam que, embora não seja regra, os negócios sustentáveis que incorporam o TBL possuem, na atualidade, uma estreita relação com a gestão empresarial (BARBIERI et al., 2010), seja pela necessidade estratégica de desenvolver inovações que tenham alguma relação com as causas socioambientais de forma complementar às questões econômicas, seja como forma de obter melhores resultados advindos desse processo.

As ISs compreendem vários contextos relacionados a processos, produtos e serviços (OKSANEN; HAUTAMAKI, 2015), dentre outras vertentes, as quais devem estar integradas a um sistema para geração de ideias, pesquisas em $P \& D$ e tecnologias que combinem a criação de valor para as empresas.

No contexto estratégico, as ISs podem mobilizar recursos, habilitar funcionários e obter informações que permitam um maior conhecimento interno e externo (GALVÃO, 2014). Assim, o potencial para geração de vantagem competitiva oriunda desse contexto oportuniza novos regulamentos sociais e ambientais e novas oportunidades de negócios, envolvendo uma ampla cooperação de vários atores para reforçar o núcleo de novas combinações de recursos e capacidades (TEECE; PISANO; SHUEN, 1997).

O contexto estratégico da sustentabilidade previsto pela TBR, no que tange à geração de vantagens relativas em termos de eficiência para as empresas, deve expressar as relações existentes entre recursos, concorrência e rentabilidade, por meio da inserção de valor, raridade, dificuldade de imitação e não substituidade pela concorrência, como forma de sustentar a vantagem competitiva (BARNEY, 1991).

Complementarmente, verifica-se a necessidade de expansão teórica da temática relacionada à IS para explicar a forma de obtenção e manutenção da vantagem competitiva, indicando as fontes e os métodos para a criação de riqueza por meio tanto da posição dos ativos ou recursos como também da geração de ideias, desenvolvimento de produtos, serviços e processos inovadores oriundos das capacidades desenvolvidas pelas empresas, ou seja, de suas CD (TEECE; PISANO, 1994; TEECE; PISANO; SHUEN, 1997).

Tal como previsto nos trabalhos relacionados ao desenvolvimento de modelos de MIS, verificou-se que a capacidade das empresas de se envolverem no processo de sustentabilidade no contexto estratégico (GALVÃO, 2014; DELAI, 2014) faz com que os melhores níveis de maturidade estejam relacionados com os melhores resultados de DFM, e que os menores níveis tenham relação com menores resultados de DFM (KNEIPP, 2016).

Dessa forma, os modelos de MIS possibilitam a gestão das ISs de forma a proporcionarem uma fonte de vantagem competitiva e melhoria no DE. Tal vantagem pode advir por meio da utilização dos recursos e capacidades que oportunizem a criação de valor aos negócios sustentáveis.

Essa pesquisa teve como limitação as poucas bases teóricas e empíricas sobre o tema relacionado as ISs para análise de suas implicações no DE, não permitindo a generalização dos resultados. Outro ponto que merece destaque diz respeito ao fato da amostra considerar um contexto regional específico, e outros trabalhos que busquem ampliar essa abordagem podem obter outros resultados. Portanto, recomenda-se a ampliação dessa pesquisa, considerando outras amostras, para que se possa avançar nessa área de conhecimento. 


\section{REFERÊNCIAS}

BARBIERI, J. C.; VASCONCELOS, I. F. G.; ANDREASSI, T.; VASCONCELOS, F. C. Inovação e Sustentabilidade: Novos Modelos e Proposições. RAE - São Paulo, v. 50, n. 2, p. 146-154, 2010.

BARNEY, J. B. Organizational Culture: Can It Be a Source of Sustained Competitive Advantage? The Academy of Management Review, v. 11, n. 3, p. 656-665, 1986.

; Firm Resources and Sustained Competitive Advantage. Journal of Management, v. 17, n. 1, p. 99-120, 1991.

; ARIKAN, A. M. The resource-based view: origins and implications. In HITT, M. A.; FREEMAN, R. E.; HARRISON, J. S. (Ed.). Handbook of strategic management. Oxford: Blackwell, 2001.

BOCKEN, N. M. P.; SHORT, S.; RANA P.; EVANS, S. A literature and practice review to develop Sustainable Business Model Archetypes. Journal of Cleaner Production, p. 1-34, 2013.

BOONS, F.; LUDEKE-FREUND, F. Business Models for Sustainable Innovation: State of the Art and Steps Towards a Research Agenda. Journal of Cleaner Production, v. 45, p. 9-19, 2013.

BOONS, F.; MONTALVO, C.; QUIST, J.; WAGNER, M. Sustainable innovation, business models and economic performance: an overview. Journal of Cleaner Production, v. 45, p. 1-8, 2013.

CHARTER, M.; CLARK, T. Sustainable Innovation: Key conclusions from Sustainable Innovation Conferences 2003-2006 organised by The Centre for Sustainable Design. SEEDA - South East England Development Agency, 2007.

CHARTER, M.; GRAY, C.; CLARK, T.; WOOLMAN, T. Review: the role of business in realising sustainable consumption and production. In: Tukker, A., Charter, M., Vezzoli, C., St $\varnothing$, E., Andersen, M.M. (Eds.). Perspectives on Radical Changes to Sustainable Consumption and Production. System Innovation for Sustainability. Greenleaf, Sheffield, p. 46-69, 2008.

CHENG, C. C. J.; YANG, C. I.; SHEU, C. The link between eco-innovation and business performance: a Taiwanese industry contexto. Journal of Cleaner Production, v. 64, p. 81-90, 2014.

COHEN, J. Statistical power analysis for the behavioural sciences. Hillside. NJ: Lawrence Earlbaum Associates, 1988.

CRONBACH, L. J. Coeficient alpha and internal structure of tests. Psychometrika, v. 16, p. 297-334, 1951.

DELAI, I. Estágios evolutivos em gestão da inovação sustentável: estudo longitudinal multicasos em empresas industriais. 221 f. Tese (Doutorado em Administração). Universidade de São Paulo - USP, Ribeirão Preto, 2014.

EISENHARDT, K. M.; MARTIN, J. A. Dynamic Capabilities: What are They? Strategic Management Journal, v. 21, p. 1105-1121, 2000.

FEDERAÇÃO DAS INDÚSTRIAS DO ESTADO DE RONDÔNIA. Disponível em: https://portal.fiero.org. br/. Acesso em 05/02/2016.

FOLLMANN, N. Modelo de Maturidade Logística para Empresas Industriais de Grande Porte. $178 \mathrm{f}$. Tese (Doutorado em Engenharia da Produção). Universidade Federal de Santa Catarina. Florianópolis, 2012. 
FOXON, T.; PEARSON, P. Overcoming barriers to innovation and diffusion of cleaner technologies: some features of a sustainable innovation policy regime. Journal of Cleaner Production, v. 16, p. 148-161, 2008.

FROEHLICH, C. O Desenvolvimento da Capacidade de Inovação para Alavancar a Sustentabilidade Empresarial: estudos de caso na Artecola e no Hospital Madre de Deus. 296 f. Tese (Doutorado em Administração). Universidade do Vale do Rio Sinos - UNISINOS-RS, São Leopoldo, 2014.

GALVÃO, H. M. Influência da Gestão Ambiental no Desempenho da Eco-invação Empresarial. 228 f. Tese (Doutorado em Administração). Universidade de São Paulo - USP, São Paulo, 2014.

GRANT, R. M. The Resource-Based Theory of Competitive Advantage: Implications for Strategy Formulation. Califórnia Management Review, p. 114-135, 1991.

GUNDAY, G.; ULUSOY, G.; KILIC, K.; ALPKAN, L. Effects of innovation types on firm performance. International Journal of Production Economics, v. 133, n. 2, p. 662-676, 2011.

HAIR, J. F.; HULT, G. T. M.; RINGLE, C. M.; SARSTEDT, M. A Primer on Partial Least Squares Structural Equation Modeling (PLS-SEM). Sage, Thousand Oaks, 2014.

HALL, J.; VREDENBURG, H. The challenges of innovating for sustainable development. MIT Sloan Management Review, v. 45, n. 1, p. 61-68, 2003.

HANSEN, E. G., GROBE-DUNKER, F., REICHWALD, R. Sustainability Innovation Cube - A Framework to Evaluate Sustainability-Oriented Innovations. International Journal of Innovation Management, v. 13, p. 683-713, 2009.

HART, S. L. A Natural-Resource-Based View of the Firm. The Academy of Management Review, v. 20, n. 4, p. 986-1014, 1995.

HART, S. L.; MILSTEIN, M. B. Creating sustainable value. Academy al Management Executive, v. 17, n. 2, p. 56-67, 2003.

HELLSTROM, T. Dimensions of Environmentally Sustainable Innovation: the Structure of EcoInnovation Concepts. Sustainable Development, v. 15, p. 148-159, 2007.

HYNDS, E. J. BRANDT, V.; BUREK, S.; JAGER, W.; KNOX, P.; PARKER, J. P.; SCWARTZ, L.; TAYLOR, J. A.; ZIETLOW, M. Maturity Model for Sustainability in New Product Development: A new assessment tool allows companies to benchmark progress toward sustainability goals and drive NPD growth. Research-Technology Management, p. 50-57, 2014.

JANG, S. H. The Offensive Framework of Resource Based View (RBV): Inhibiting Others from Pursuing Their Own Values. Journal of Management and Strategy. v. 4, n. 1, p, 62-69, 2013.

KEMP, R.; PEARSON P. Measuring eco-innovation. Final report of the project Measuring EcoInnovation; Maastricht (The Netherlands), 2007.

KNEIPP, J. M. Gestão Estratégica da Inovação Sustentável e sua Relação com o Modelo de Negócios e o Desempenho Empresarial. 188f. Tese (Doutorado em Administração). Universidade Federal de Santa Maria - UFSM-RS, Santa Maria, 2016.

LEE, L.; PETTER, S.; FAYARD, D.; ROBINSON, S. (2011). On the use of partial least squares path modeling in accounting research. International Journal of Accounting Information Systems, v. 12, n. 4, p. 305-328, 2011. 
LOCKETT, A. Edith Penrose's legacy to the resource-based view. Managerial and Decision Economics, v. 26, n. 2, p. 83-98, 2005.

LOPEZ-VALEIRAS, E.; GOMEZ-CONDE, J.; NARANJO-GIL, D. Sustainable Innovation, Management Accounting and Control Systems, and International Performance. Sustainability, v. 7, p. 34793492, 2015.

MCKELVIE, A.; DAVIDSON, P. From Resource Base to Dynamic Capabilities: An Investigation of New Firms. British Journal of Management, v. 20, p. 1-41, 2009.

NUNNALlY, J. C.; BERSTEIN, I. H. Pyschometric theory - 3 ed. New York: MacGraw-Hill, 1994.

OKSANEN, K.; HAUTAMAKI, A. Sustainable Innovation: A Competitive Advantage for Innovation Ecosystems. Technology Innovation Management Review, v. 5, n. 10, p. 24-30, 2015.

PELHAM, A. M.; WILSON, D. T. A Longitudinal Study of the Impact of Market Structure, Firm Structure, Strategy, and Market Orientation Culture on Dimensions of Small-Firm Performance. Journal of the Academy of Marketing Science, v. 24, n. 1. p. 27-43, 1996.

PLANO AMAZÔNIA SUSTENTÁVEL (PAS): diretrizes para o desenvolvimento sustentável da Amazônia brasileira. Brasília, Editorial Abaré, MMA, 2008.

RENNINGS, K. Redefining Innovation: eco-innovation research and the contribution from ecological economics. Ecological Economics, v. 32, p. 319-332, 2000.

RINGLE, C.; M.; SILVA, D.; BIDO, D. S. Modelagem de equações estruturais com utilização do SmartPLS. REMark, v. 13, n. 2, p. 54, 2014.

RUSSO, M. V.; FOUTS, P. A. A Resource-Based Perspective on Corporate Environmental Performance and Profitability. The Academy of Management Journal, v. 40, n. 3, p. 534-559, 1997.

SCHALTEGGER. S.; LÜDEKE-FREUND, F.; HANSEN, E. G. Business Cases for Sustainability and the Role of Business Model Innovation: Developing a Conceptual Framework. Center for Sustainability Management (CSM), Leuphana. University of Lueneburg Scharnhorststr, p. 1-32, 2011.

SHARMA, S.; VREDENBURG, H. Proactive Corporate Environmental Strategy and the Development of Competitively Valuable Organizational Capabilities. Strategic Management Journal, v. 19, p. 729-753, 1998.

SHRIVASTAVA, P.; HART, S. Creating Sustainable Corporations. Business Strategy and the Environment, v. 4, p. 154-165, 1995.

STREINER, D. L. Being inconsistent about consistency: when coefficient alpha does and doesn't matter. Journal of Personality Assessment, v. 80, 2003, p. 217-222.

TEECE, D. J.; PISANO, G. The Dynamic Capabilities of Firms: an Introduction. Industrial and Corporate Charge, v. 3, n. 3, p. 537-556, 1994.

TEECE, D. J.; PISANO, G.; SHUEN, A. Dynamic Capabilities and Strategic Management. Strategic Management Journal, v. 18, n. 7, p. 509-533, 1997.

WERNERFELT, B. Resource-Based View of the Firm. Strategic Management Journal, v. 5, n. 2, p. 171-180, 1884. 